\title{
The Impact of Anxiety on Processing Efficiency: Implications for the Attentional Control Theory
}

\author{
Wong, I. $\mathrm{Y}^{1^{*}}$, Mahar, D. ${ }^{2}$, Titchener, K. and Freeman, $\mathrm{J}^{1}$ \\ ${ }^{1}$ Centre for Accident Research and Road Safety-Queensland, 130 Victoria Park Road, Kelvin Grove 4059, Australia \\ ${ }^{2}$ School of Psychology and Counselling, Queensland University of Technology, Australia
}

\begin{abstract}
The Attentional Control Theory (ACT) proposes that high-anxious individuals maintain performance effectiveness (accuracy) at the expense of processing efficiency (response time), in particular, the two central executive functions of inhibition and shifting. In contrast, research has generally failed to consider the third executive function which relates to the function of updating. In the current study, seventy-five participants completed the Parametric Go/NoGo and $n$-back tasks, as well as the State-Trait Anxiety Inventory in order to explore the effects of anxiety on attention. Results indicated that anxiety lead to decay in processing efficiency, but not in performance effectiveness, across all three Central Executive functions (inhibition, set-shifting and updating). Interestingly, participants with high levels of trait anxiety also exhibited impaired performance effectiveness on the $n$-back task designed to measure the updating function. Findings are discussed in relation to developing a new model of ACT that also includes the role of preattentive processes and dual-task coordination when exploring the effects of anxiety on task performance.
\end{abstract}

Keywords: Central Executive, Attentional Control, Trait Anxiety, Processing Efficiency.

\section{INTRODUCTION}

Much empirical attention has been devoted to the influence of anxiety on task performance across a variety of domains, such as test anxiety $[1,2]$ and sports performance $[3,4]$. Behavioural evidence has emerged to indicate that high levels of anxiety are associated with decreased task performance, especially on tasks that are mentally demanding $[5,6]$. While a number of theories have been developed in an attempt to explain the complex relationship between anxiety and task performance, the Attentional Control Theory (ACT) remains the only theory that can account for both the facilitative and debilitative effect of anxiety reported in the literature [4]. ACT is considered a major extension of the Processing Efficiency Theory (PET), developed by Eysenck and Calvo [7], which proposes that worry (the main cognitive component of anxiety) affects task performance through pre-empting some of the processing and storage capacity of the Working Memory system. As a result, anxious individuals experience decreased performance on tasks that place high demands upon Working Memory. Eysenck and Calvo [7] proposed that worry also leads to an increase in motivation in order to preserve task performance level, resulting in the allocation of additional processing resources (effort) and the deployment of processing activities (compensatory strategy) aimed at improving task performance.

*Address correspondence to this author at the Centre for Accident Research and road Safety-Queensland; Tel: 61731387698; Fax: 61731380111;

E-mail: ides.wong@qut.edu.au
While PET received encouraging support from many behavioural studies using various cognitive and motor tasks $[4,8-10]$, there are several limitations of the theory in accounting for the effect of anxiety on cognitive performance. For example, Eysenck et al. [11] highlighted PET's lack of precision in predicting the effects of anxiety. As a result, Attentional Control Theory (ACT) was developed as a significant advancement to the PET. Specifically, ACT builds upon the strength of PET by incorporating its emphasis on processing efficiency and performance effectiveness, and addresses previous limitations through extending the scope of the theory to include a more precise explanation of the effects of anxiety on the Central Executive functions.

A central tenet of ACT is the prediction that anxiety impacts task performance through its deleterious effects on attentional control, a crucial component of the Central Executive [12]. This is because anxiety is often experienced when a current goal is under threat. In order to preserve goal attainment, individuals would allocate attentional resources to the identification and processing of the source of the potential threat [11]. This assumption is supported by findings that anxious individuals demonstrate attentional bias towards threat-related stimuli in the presence of neutral ones [12-14]. A further assumption of ACT is that anxiety can cause impairment in attentional control without the presence of threat-related, task-irrelevant stimuli. Eysenck et al. [11] postulated that when an individual feels threatened, it could be counter-productive to continue directing high levels of attentional resources towards a specific location. Rather, the optimal strategy would be to distribute attentional resources 
widely, subsequently reducing the amount of attention available towards the current task [11].

The above theoretical assumptions of ACT are based on the premise that the allocation of attentional resources is governed by the interaction of two attentional systems proposed by Corbetta and Shulman [15]: the Goal-Driven Attentional System (GDAS) and the Stimulus-Driven Attentional System (SDAS). GDAS exemplifies top-down attentional control, and is shaped by the individual's current goals, knowledge and expectations; whereas SDAS exemplifies bottom-up attentional control, and is influenced by salient environmental stimuli [12]. Corbetta and Shulman proposed that an optimal balance between these two systems is achieved through bidirectional interaction of the two systems, and that disturbance of this balance would result in decreased attentional control. Within ACT, anxiety disrupts the balance between these two systems, with increased anxiety leading to a decreased influence of GDAS and an increased influence of the SDAS through preferential processing to (and inhibit disengaging from) task-irrelevant threat-related stimuli.

Based on lower level functions of the Central Executive component that have previously been proposed [16-18], three major Central Executive functions have been identified which are inhibition, set-shifting and updating. Inhibition involves the use of attentional control in a restraining manner to prevent attentional resources being distributed to task-irrelevant stimuli [11]. The shifting function identified by Miyake [18] is also related to Attentional Control Theory, because it involves the use of attentional control in a positive manner in terms of shifting attentional resources to remain focused on task-relevant stimuli. However, Eysenck et al. [11] stated that the influence of anxiety on the shifting function is less clear and should be further explored. Results from subsequent studies by Ansari, Derakshan and Richards [19] and Ansari and Derakshan [20] using a mixed antissaccade paradigm, in which participants were occasionally instructed to make an intentional saccade to the direction opposite the stimulus, appeared to provide support for this hypothesis. Reduced inhibition was also observed through the use of task-irrelevant distracters, providing further support for the notion of impoverished inhibition due to anxiety [21].

Importantly, no existing experiments have explored the effects of anxiety on the updating function as predicted by ACT. Behaviourally, the effects of anxiety on the updating function has yielded inconsistent results [11]. According to Derakshan and Eysenck [12], because the updating function involves memory instead of attention, it is not directly affected by anxiety. Thus, the most important assumption of ACT is that anxiety negatively affects the processing efficiency of the set-shifting and inhibition functions, and that anxiety is presumed to only influence the updating function under stressful conditions. However, to the author's knowledge, this assumption of ACT has not been empirically tested. According to a recent review of the theory by Berggren and Derakshan [22], studies exploring the effects of anxiety on the monitoring and updating of working memory are lacking, such as when participants have to maintain mental representations in memory and must constantly update that information. Thus, the current study intended to further explore whether anxiety also impacts upon the updating function using the $n$-back task, which requires participants to constantly maintain and update mental representations within the working memory.

ACT has received encouraging empirical support using mixed antisaccade paradigms [19, 23], which has demonstrated the effect of anxiety on task switching and inhibition to be consistent with the predictions of ACT. However, a criticism of the current anxiety-cognition literature is that many studies have employed only one single cognitive task in their design. More specifically, Shackman et al. [24] proposes that multiple tasks should be employed in order to allow for accurate inferences about the specificity of any observed affective modulation. This is because if only a single cognitive task is used and the manipulation of anxiety results in a significant effect on task performance, it would be difficult to infer the specificity of the effect [24]. This limitation is especially relevant to studies on Attentional Control Theory, as the inhibition, shifting, and updating functions are partially interdependent [11]. This poses a significant challenge in the interpretation of studies that claim to be supportive of ACT. To date, none of the published studies that provided experimental support for ACT have employed multiple cognitive tasks to assess changes in all three Central Executive functions resulting from the induction of anxiety. Additionally, it has been proposed that research needs to be extended and utilise cognitive tasks that are psychometrically equivalent and possess a well-characterised cognitive structure [24]. More specifically, using cognitive tasks that have a wellunderstood functional neuroanatomy character, such as the Go/No-Go task will allow researchers to infer the plausible loci of any observed Anxiety by Cognition interactions [24]. This limitation is pertinent to the experimental studies of ACT due to the use of the antisaccade task as the measure of cognitive performance. While saccadic performance may reflect an individual's level of attentional control, it can also reflect behavioural inhibition that may not be related to attention, such as control of eye movements [19]. Finally, studies that used the PGNG task typically only adopt accuracy rate (percentage of correct set-shifting/inhibitory trials) as a measure of performance effectiveness and thus fail to account for other response types (e.g. false alarms). Taken together, these limitations restrict the interpretations that can be drawn from the results of the current body of research on ACT, although it is noted that that the theoretical framework remains popular as it is the only one that considers the distinction between performance effectiveness and processing efficiency.

The current study aimed to utilise more compatible cognitive measures (go/no-go task and $n$-back task) designed to specifically test the effect of anxiety on the updating function, as well as simultaneously gauge the effect of anxiety on each of the Central Executive functions proposed by Miyake et al., [18]. Participants' trait anxiety levels were expected to negatively impact their levels of processing efficiency on their Central Executive functions of setshifting, inhibition and updating, indexed by their averaged response time in the Go/No-Go task (set-shifting and inhibition) and the numeric $n$-back task (updating). The performance effectiveness of these functions, as indexed by 
the values of $d^{\prime}$ of the correct inhibitory set (inhibition), correct target set (shifting) and the $d$ ' values in the numeric $n$-back task (updating) was expected to be similar across high and low-trait anxious participants.

In sum, four hypotheses were proposed. First, consistent with findings of previous studies that used the PGNG and $n$ back tasks [25, 28], both high and low anxious participants would demonstrate increased response time as the task increased in difficulty. Secondly, under ACT, compared to low anxious participants, decay in processing efficiency (i.e. longer RT) due to task difficulty would be greater for high anxious participants on both the PGNG and $n$-back tasks. Thirdly, both high and low trait anxious participants' levels of accuracy (i.e. values of $d^{\prime}$ ) were expected to deteriorate with increased task difficulty. The fourth hypothesis was that according to ACT, anxiety would impair processing efficiency (i.e. RT) to a greater extent than performance effectiveness (i.e. accuracy).

\section{METHOD}

\section{Participants}

Seventy-five university students (53 female) participated in the experiment. Participants ranged from 17 to 47 years old $(M=24.45, S D=7.77)$. All participants received entry to a prize draw of one of two $\$ 100.00$ shopping vouchers upon completion of the experiment.

\section{Design}

The study adopted a quasi-experimental between-subjects design. The independent variable was levels of trait anxiety, operationalised across two levels of high $(\geq 38)$ and low anxiety $(\leq 37)$ using scores obtained from the STAI (Form Y-2). The first set of dependent variables was processing efficiency, indexed by the averaged response time of correct trials at each level of the PGNG and $n$-back tasks. The second set of dependent variables was performance effectiveness, indexed by stimuli sensitivity $\left(d^{\prime}\right)$, determined by the proportion of hits and false alarms, for the set-shifting and inhibition function from the PGNG task, and the updating function from the $n$-back task.

\section{Materials and Procedure}

Upon arrival at the testing room, each participant was assigned a computer equipped with the PGNG and $n$-back software. Participants were then instructed to provide informed written consent, and complete Form Y-2 of the State-Trait Anxiety Inventory (STAI; [26]). The researcher then explained the format of the experimental trial and provided detailed instruction of the experiment. The Parametric Go/No-Go and $n$-back tasks were presented in counterbalanced order, separated by a short break between the two tasks. The experiment began with screen instructions for the according task on participants' computer screens.

\section{Parametric Go/No-Go task}

The Parametric Go/No-Go (PGNG) task, designed to measure the inhibition and shifting functions, is described in detail elsewhere [27]. Briefly, the PGNG consisted of three levels of difficulty, completed in order of ascending difficulty. Participants were shown a series of letters on the computer screen for $1 \mathrm{~s}$ per letter with no interstimulus interval, and were required to click on the 'Target Present' button whenever the presented letter met the specified rule of the trial. For the first 210 trials (i.e. Level 1) of the PGNG task, participants had to click 'Target Present" whenever the letter X, Y, or Z was presented. For the second 210 trials (i.e. Level 2), participants had to respond when the letter presented was either $\mathrm{X}$ or $\mathrm{Y}$, in a non-repeating order. In other words, participants had to click the 'Target Present' button only if the last responded letter was different to the current letter. For the third 210 trials (i.e. Level 3), participants had to respond to the target set X, Y, or Z using the same non-repeating rule as level 2. For example, if the last responded letter was ' $\mathrm{X}$ ', then participants had to click 'Target Present' as soon as they were presented ' $\mathrm{Y}$ ' or ' $\mathrm{Z}$ ', but had to inhibit from responding to ' $\mathrm{X}$ '.

For the purpose of testing ACT in the current study, participants' performance effectiveness was based on the measure of stimuli sensitivity $\left(d^{\prime}\right)$ to take into account the proportion of false alarms as well as hits, providing a more comprehensive understanding of participants' response style. A 'Hit' required the participants correctly clicked on the 'Target Present' button when the presented stimuli met the specified rule of the trial, whereas a 'False Alarm' occurred when the presented stimuli did not meet the specified rule of the trial and the participant incorrectly clicked 'Target Present'.

Each level of the PGNG task consisted of 210 trials, with the initial 5 trials as practice trials. Each level consisted of 28 hit trials for the set shifting function and 15 hit trials for the inhibition function, with the remaining trials as rejection trials for each function. The sequence of hit and miss trials were the same across all participants.

\section{N-Back Task}

The $n$-back task [28] was used to gauge participants' updating function. It required respondents to consciously follow a series of numbers and respond when they saw a number that was presented at the previous trial (1-back), after one intervening number (2-back), or after two intervening numbers (3-back). Numbers were used in this task to prevent the carry-over effect associated with the use of letters in the PGNG task. Similar to the PGNG task, the three levels of the task were presented in order of ascending difficulty. For all three levels, a serial stream of single digits (1 to 9) was presented on the computer screen. Each number was presented for $500 \mathrm{~ms}$ with no interstimulus interval.

Participants were required to click the 'Target Present' button whenever the presented digit met the specified rule of the trial. For the first 60 trials, participants were asked to click the 'Target Present' button whenever the digit displayed was the same as the one shown in the previous trial (e.g. 3, 3). For the second 60 trials, participants were required to click the 'Target Present' button whenever the digit displayed on the screen was identical to the one presented two trials earlier (e.g. 3, 2, 3). For the third 60 trials, participants were required to respond whenever the onscreen digit was same as the one displayed three trials earlier (e.g. 3, 2, 6, 3). 
Similar to the PGNG task, stimuli sensitivity ( $d$ ') was used to measure the performance effectiveness of the participant's updating function; while the processing efficiency of participant's updating function was indexed by their averaged reaction time for the correct target trials. Each level of the $n$-back task consisted of five practice trials, followed by 55 target trials. Ten target trials of each level were hit trials, while the remaining trials for each level were rejection trials. The sequence of hit and miss trials was the same across all participants.

Upon conclusion of both the PGNG task and the numeric $n$-back task, participants were required to complete the remaining questionnaires, which concluded the experiment. Participants were then debriefed and dismissed.

\section{Results}

Participants scores on the STAI as well as their processing efficiency (RT) and performance effectiveness (accuracy, indexed by $d^{\prime}$ ) on the PGNG and $n$-back tasks were analysed. Histograms revealed one outlying case, with a score of zero on all levels of both the PGNG and $n$-back tasks, which was excluded from analysis. The median trait anxiety score for the sample was 37.5. Similar to Ansari et al. (2008) and Derakshan et al. (2009), median splits on the trait anxiety questionnaire was used to produce high and low anxiety group classifications. Subsequent statistical analysis considered participants who obtained trait anxiety scores of 37 or below as low anxious $(N=37, M=30.51, S D=4.47)$, and 38 or above as high anxious $(N=37, M=47.22, S D=8.55)$.

\section{Central Executive Functions}

A series of mixed model ANOVAs were conducted to assess the effects of trait anxiety on performance effectiveness $\left(d^{\prime}\right)$ and efficiency (RT) across each of the
Central Executive functions of set-shifting, inhibition and updating. As the PGNG task simultaneously measures the set-shifting and inhibition functions, a combined response time was used for both functions.

\section{Response Times for Set-Shifting and Inhibition Functions}

As illustrated in Fig. (1), task difficulty had a significant main effect upon participants' response times for the SetShifting and Inhibition functions of the PGNG task, indicating that participants took longer to respond as the task increased in difficulty, $F(2,71)=64.74, p<.001, \eta_{p}{ }^{2}=.65$. Specifically, Level 3 elicited longer response times than either Level $1, F(1,72)=80.02, p<.001, \eta_{p}{ }^{2}=.53$, and Level $2, F(1,72)=112.70, p<.001, \eta_{p}{ }^{2}=.61$. A significant main effect of anxiety was also found, $F(1,72)=13.04, p=.001$, $\eta_{p}{ }^{2}=.15$. On average, high trait anxious participants took $45.04 \mathrm{~ms}$ (95\% CI from $20.39 \mathrm{~ms}$ to $69.42 \mathrm{~ms}$ ) longer to respond to the stimuli than did low trait anxious participants. There was also a significant interaction between trait anxiety and task difficulty on response times, $F(2,71)=3.45, p$ $=.037, \eta_{p}{ }^{2}=.09$; indicating that the effect of task difficulty on response time varied depending on the level of trait anxiety. Specifically, high trait anxious participants took significantly longer than low anxious participants to respond to task stimuli in the more mentally demanding Level $2, F(1,72)=$ $13.50, p=<.001, \eta_{p}{ }^{2}=.16$, and Level $3, F(1,72)=15.80, p$ $<.001, \eta_{p}{ }^{2}=.18$, suggesting that trait anxiety accelerates the increase in response time with increased task difficulty.

\section{Set-Shifting}

Accuracy. Task difficulty had a significant main effect on the accuracy rate of the set-shifting trials, $F(2,71)=15.39$, $\mathrm{p}<.001, \eta_{\mathrm{p}}{ }^{2}=.30$ (see Fig. 2). Participants experienced a rapid found for trait anxiety on response accuracy, $F(1,72)=.02$, $\mathrm{p}=.89, \eta_{\mathrm{p}}{ }^{2}<.001$. Finally, trait anxiety and task difficulty

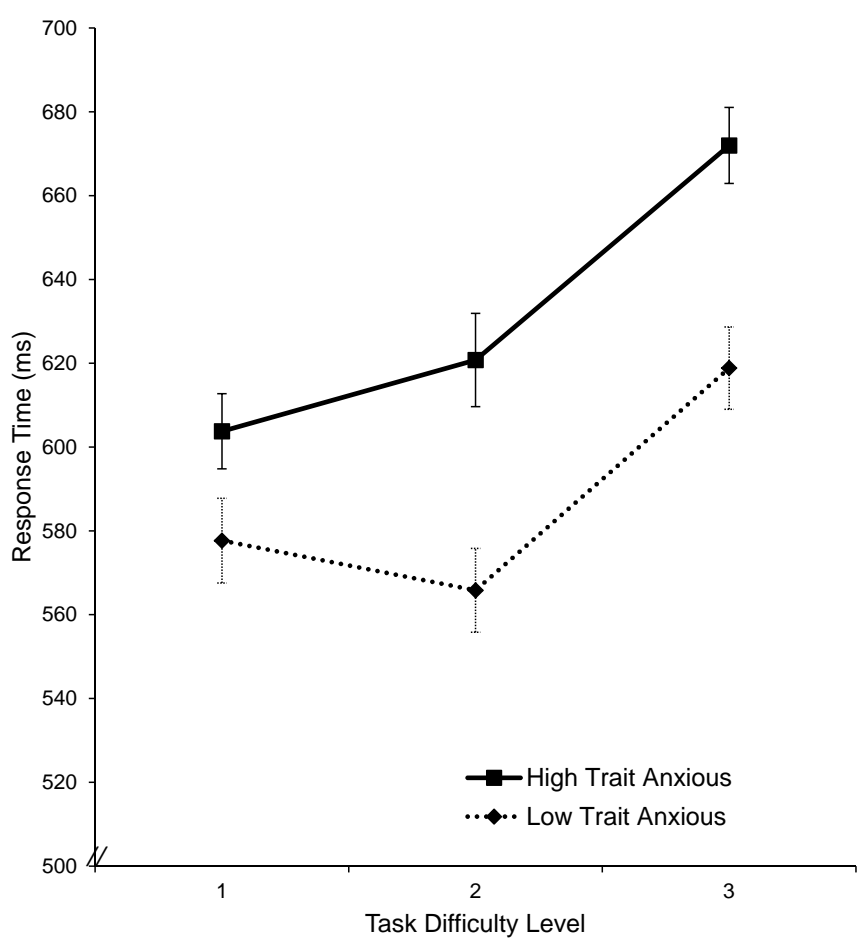

Fig. (1). Mean response times (ms) for low- and high-anxious individuals comparing task difficulty levels for the set-shifting and inhibition targets on the PGNG task (error bars $=\mathrm{SE}$ ). 


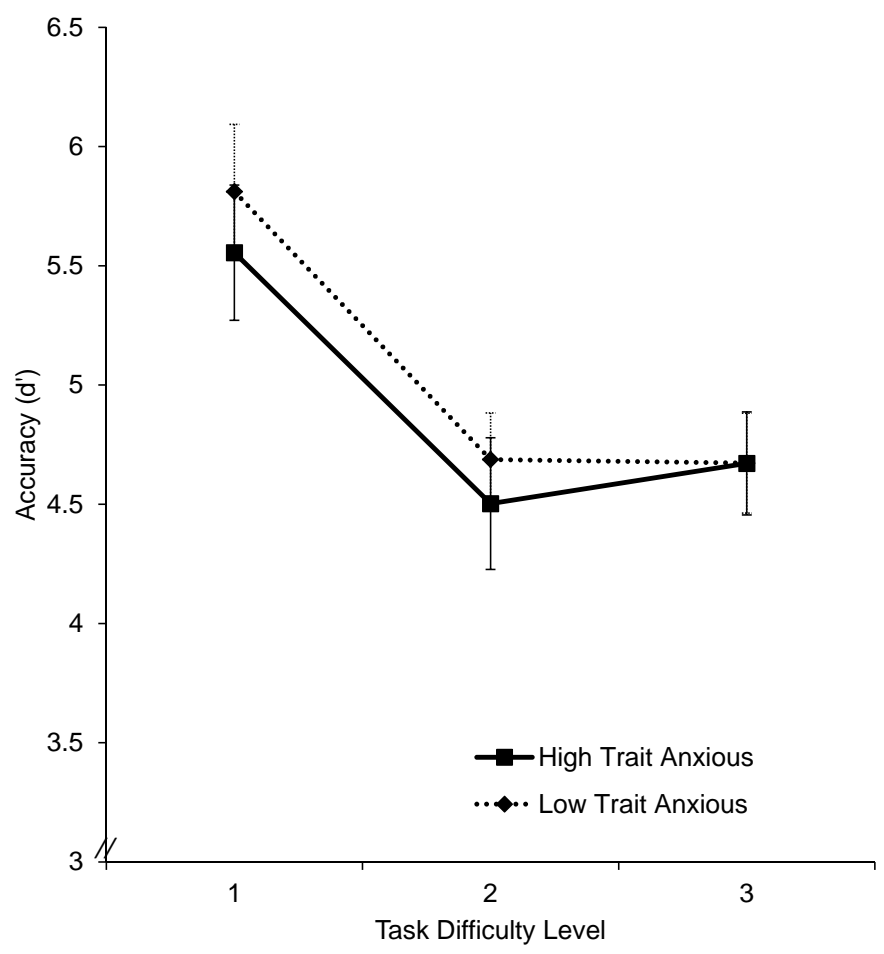

Fig. (2). Mean response accuracy (d') for low- and high-anxious individuals comparing task difficulty levels for the set-shifting targets on the PGNG task (error bars $=\mathrm{SE})$.

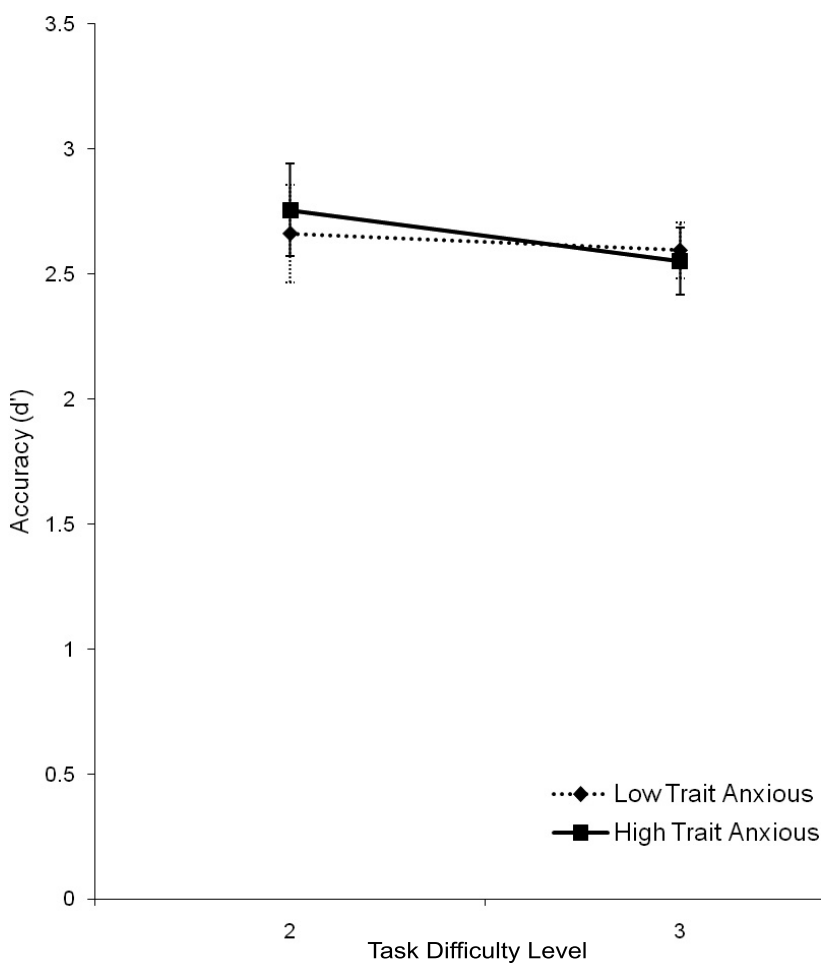

Fig. (3). Mean response accuracy (d') for low- and high-anxious individuals comparing task difficulty levels for the inhibition targets on the PGNG task (error bars $=\mathrm{SE})$

decay in accuracy between Level 1 and Level $2, \mathrm{~F}(1,72)=$ $21.65, \mathrm{p}<.001, \eta_{\mathrm{p}}{ }^{2}=.23$, while participants' stimuli sensitivity was similar between Levels 2 and $3, \mathrm{~F}(1,72)=.17, \mathrm{p}=.69, \eta_{\mathrm{p}}{ }^{2}=$ .002. Participants' levels of trait anxiety had no main effect on accuracy rates, $\mathrm{F}(1,72)=.33, \mathrm{p}=.57, \eta_{\mathrm{p}}{ }^{2}=.005$. Finally, task difficulty and trait anxiety had no interaction effect on response accuracy, $\mathrm{F}(2,71)=.20, \mathrm{p}=.82, \eta_{\mathrm{p}}^{2}=.005$.

\section{Inhibition}

Accuracy. As expected, task difficulty had no significant main effect on response accuracy, $F(1,72)=1.38, p=.25$. $\eta_{\mathrm{p}}{ }^{2}=.02$ (see Fig. 3). Further, no significant main effect was had no significant interaction effect on response accuracy, $\mathrm{F}$ $(1,72)=.36, \mathrm{p}=.55, \eta_{\mathrm{p}}{ }^{2}=.005$. 


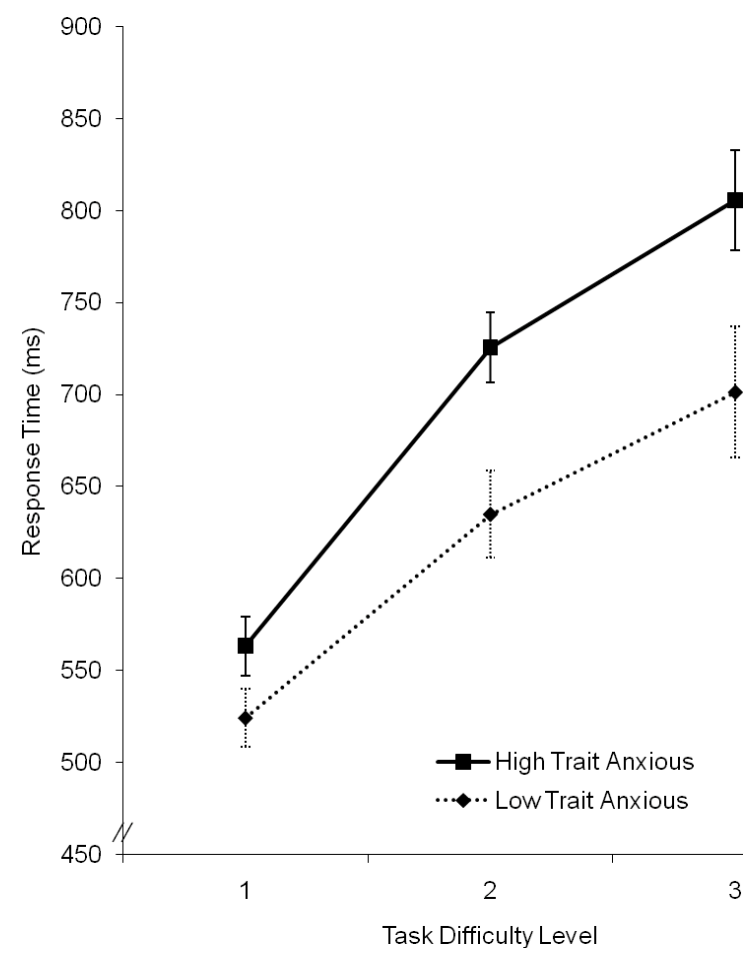

Fig. (4). Mean response times (ms) for low- and high-anxious individuals comparing task difficulty levels for the updating (n-back) task (error bars $=\mathrm{SE})$.

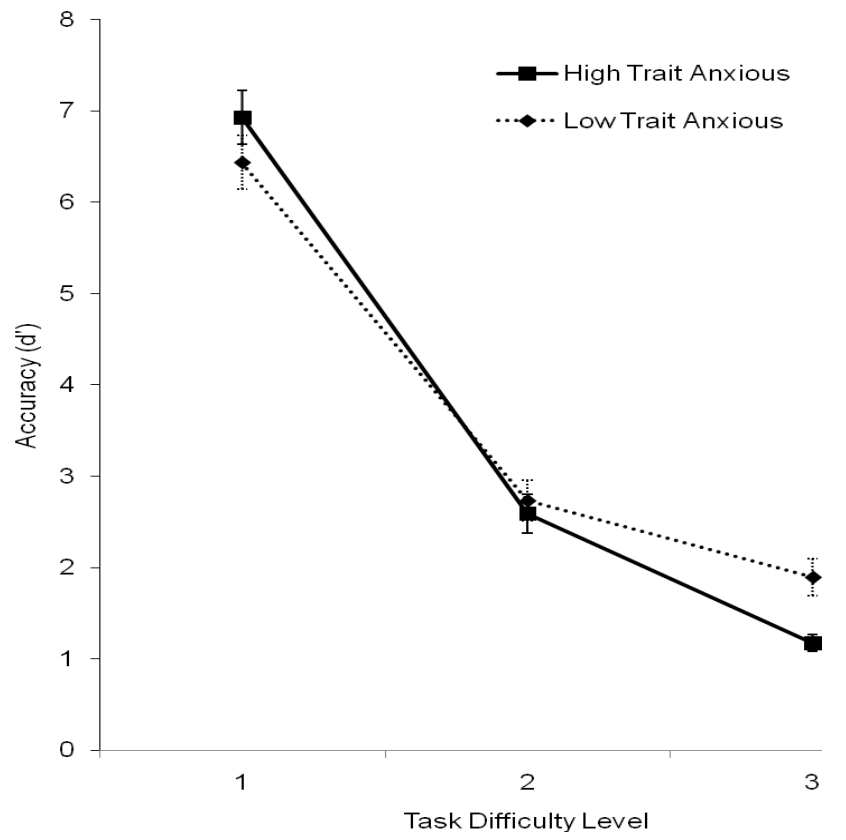

Fig. (5). Mean response accuracy (d') for low- and high-anxious individuals comparing task difficulty levels on the updating (n-back) task $($ error bars $=\mathrm{SE})$.

\section{Updating}

Response time. As shown in Fig. (4), increasing task difficulty had a significant main effect upon participants' response times for the n-back task, $F(2,71)=47.69, p$ $<.001, \eta_{\mathrm{p}}{ }^{2}=.57$. Specifically, the most mentally demanding 3-back level elicited longer response times than both the 1back level, $\mathrm{F}(1,72)=87.76, \mathrm{p}<.001, \eta_{\mathrm{p}}{ }^{2}=.55$, and the 2 back level, $\mathrm{F}(1,72)=17.65, \mathrm{p}<.001, \eta_{\mathrm{p}}{ }^{2}=.20$. Trait anxiety had a significant main effect on response time, F (1, $72)=9.06, p=.004, \eta_{p}{ }^{2}=.11$. Compared to low trait-anxious participants, participants who reported high trait anxiety took, on average, $77.45 \mathrm{~ms}$, with a $95 \%$ confidence interval ranging from $26.15 \mathrm{~ms}$ to $128.76 \mathrm{~ms}$, longer to respond to task stimuli across the 3 difficulty levels.

Although Fig. (4) indicates that, compared to low trait anxious participants, high trait anxious participants demonstrated longer response times with increased task difficulty, the interaction between task difficulty and trait anxiety was found to be non-significant, $F(2,71)=1.37, p=$ $.26, \eta_{p}{ }^{2}=.04$, with an observed power of .29. However, 
compared to low anxious participants, high anxious participants recorded significantly longer RT in level 2, $F$ (1, $72)=8.91, p=.001, \eta_{p}{ }^{2}=.11$, and level $3, F(1,72)=5.45$, $p=.02, \eta_{p}{ }^{2}=.07$, but not in level $1, F(1,72)=3.05, p=.09$, $\eta_{p}{ }^{2}=.04$.

Accuracy. Increasing difficulty of the n-back task had a substantial effect upon participants' accuracy rate, F (2, 71) $=198.60, \mathrm{p}<.001, \eta_{\mathrm{p}}{ }^{2}=.85$. As illustrated in Fig. (5), participants were significantly less accurate in Level 3 than Level 1, F $(1,72)=401.99, \mathrm{p}<.001, \eta_{\mathrm{p}}{ }^{2}=.85$, and Level 2, $\mathrm{F}(1,72)=41.60, \mathrm{p}<.001, \eta_{\mathrm{p}}{ }^{2}=.37$. Trait anxiety had no main effect on accuracy, $F(1,72)=.44, p=.51, \eta_{p}{ }^{2}=.006$. Finally, task difficulty and trait anxiety had a significant interaction effect upon participants' accuracy rates, $\mathrm{F}(2,71)$ $=3.14, \mathrm{p}=.05, \eta_{\mathrm{p}}{ }^{2}=.08$. Specifically, compared to low-trait anxious participants, high trait anxious participants were significantly less accurate in their responses in Level 3 of the n-back task, $\mathrm{F}(1,72)=10.58, \mathrm{p}=.002, \eta_{\mathrm{p}}{ }^{2}=.13$.

\section{DISCUSSION}

The present study aimed to establish whether high anxious individuals maintain performance effectiveness (accuracy, as indexed by d') at the expense of processing efficiency (response time), using cognitive tasks that exert a high demand on Central Executive functions. The current study is one of the first to examine the predictions of the Attentional Control Theory for the updating function and to simultaneously test the effects of anxiety on all three central executive functions under the premise of ACT . Overall, the results from the cognitive tasks indicated that anxiety lead to decay in processing efficiency, but not in performance effectiveness, across all three Central Executive functions (inhibition, set-shifting and updating).

\section{Research Findings}

Congruent with previous studies that used the PGNG [26] and $n$-back tasks [25], both high and low anxious participants would demonstrate decreased processing efficiency (i.e. longer RT) with increased task difficulty in both the PGNG and $n$-back tasks. The second hypothesis, that the decay in processing efficiency (i.e. longer RT) due to task difficulty would be greater for high anxious participants, was also supported in both the PGNG and $n$-back tasks. This is consistent with ACT as it proposes that anxiety disrupts the balance between the Goal-Driven Attentional System (GDAS) and the Stimulus-Driven Attentional System (SDAS), leading to a decrease in processing efficiency on the attentional control functions of set-shifting, inhibition and updating [11]. This finding is also in line with previous studies that investigated the effects of trait anxiety on response times in a range of cognitive tasks [19, 23] (e.g. Ansari et al., 2008; Derakshan et al., 2009).

Further, participants' performance effectiveness deteriorated with increased task difficult. Participants' levels of accuracy demonstrated the expected decline with increased task difficulty across all but the inhibition function. This is not surprising given participants' accuracy rates were similar across the more difficult levels of the setshifting function, suggesting a less mentally demanding task level, similar to Level 1 of the set-shifting function, is required to gauge the inhibitory function.
Finally, anxiety impaired processing efficiency to a greater extent than performance effectiveness. The decreases in performance effectiveness due to increased task difficulty were similar across both high and low anxious participants. Compared to low anxious participants, high anxious participants demonstrated a more rapid decrease in their accuracy rate in the most demanding level of the $n$-back task. This is consistent with the prediction of ACT [11], that anxiety impairs processing efficiency (and sometimes effectiveness) of the Updating function only under demanding situations. While this finding is in line with those of Darke [29] and Calvo, Ramos, and Estevez [30], who compared the performance of high and low anxious participants on reading-span tasks, it contradicts Sorg and Whitney's [31] findings of no-clear differences on the Updating task between high and low anxious participants, possibly due to the undemanding nature of the tasks used in Sorg and Whitney's study.

\section{Considerations of Research Findings}

While ACT has received substantial support both from past research and from the present results, it does not appear to take account of the distinction between attentive and preattentive processes. According to Wolfe, Treisman, and Horowitz [32], successful theories of attention must consider the role of preattentive processes. Neisser [33] pioneered the theoretical distinction between controlled (i.e. attentive) and automatic (i.e. preattentive) processes of attention. Controlled processes are operated sequentially, require attention and have a limited capacity. Whereas automatic processes are welllearned behavioural sequences automatically triggered by environmental stimuli, do not require conscious attention and can operate in parallel with other processes [34]. Further, Schneider and Schiffrin demonstrated that, given sufficient practice, tasks which require controlled attention may become automatic.

Based on observation of mental lapses, Norman and Shallice [35] expanded on the preattentive/attentive distinction, and proposed that ongoing actions (e.g. driving) are typically carried out through a process of contention scheduling. According to Norman and Shallice, preattentive processes would result in attentional lapses if left entirely to their own devices. As a result, an automatic conflict resolution process, namely contention scheduling, is required to prioritise actions based on the strength of the stimuli and individuals' expectations, knowledge and goals. An example of contention scheduling is that most drivers have experienced driving while preoccupied with other thoughts. Despite having no clear recollection of the journey travelled, they would have successfully negotiated winding roads and driving obstacles. In addition, Norman and Shallice proposed that in situations where automatic contention scheduling becomes inadequate to preserve task performance, the supervisory attentional system can intervene to take over conscious attentional control, through priming or inhibiting certain actions ${ }^{1}$.

Perhaps the most direct evidence for including the role of contention scheduling in ACT is the converging support of

\footnotetext{
1 The supervisory attentional system (SAS) was later adopted by Baddeley as a framework for the Central Executive of the Working Memory model (Baddeley, 2007), also the primary focus of the present study.
} 
its resemblance to dual-task coordination and the importance of dual-task coordination on the Central Executive. According to Baddeley [16], dual-task coordination is considered a major Central Executive function. Collette and Van der Linden's [36] review of neuroimaging studies concluded that along with inhibition, set-shifting and updating, dual-task coordination should be considered as a function of the Central Executive system. Baddeley's position regarding the role of dual-task coordination is further supported by neuropsychological studies that found, when compared to performance decay on individual tasks, patients with suspected executive function deficits, such as frontal lobe lesions, traumatic brain injury, Parkinson's and Alzheimer's disease, exhibited disproportionately larger dual-task decrements [16]. Conversely, Baddeley, Della Sala, Gray, Papagno, and Spinnler [38], Fournier-Vicente, Larigauderie, and Gaonac'h [37-39], McDowell, Whyte, and D'Esposito [40], and Miyake et al. [18] reported no correlation between performance on dual-task coordination measures and performance on any of the storage-andprocessing Central Executive functions (updating, setshifting and inhibition). The lack of correlation between performance on dual-task coordination and the inhibition, set-shifting and updating functions led Miyake et al. [18] to exclude dual-task coordination from their list of Central Executive functions.

In an attempt to reconcile the conflicting findings regarding the relationship between dual-task coordination and the traditional Central Executive functions, FournierVicente et al. [39] proposed that dual-task coordination may be a Central Executive function that taps different aspects of executive functioning independent of the three functions of updating, set-shifting and inhibition. It is proposed here that the conflicting findings of dual-task coordination can be reconciled by interpreting it as a function of contention scheduling, modulating the influence of the Goal-Directed and Stimulus-Driven Attentional Control systems. This interpretation is in line with findings from neuroimaging studies which have reported dual-task performance results in greater recruitment of brain regions already activated by component tasks, rather than activation of a novel region [37]. The assumption of dual-task coordination as a function of contention scheduling (the interaction between GoalDirected and Stimulus-Driven Attentional Systems) is further supported by the low test-retest reliabilities reported by Baddeley et al. [41] and Fournier-Vicente et al. [39]. This is because within the proposed model, dual-task coordination results from the interaction of individuals' current expectations, knowledge and goals and the strength of the salient environmental stimuli, thus it should only reflect current situations.

\section{Future Directions}

According to Derakshan and Eysenck [12], the need of more "process-pure" tasks that reflect primarily a single underlying Central Executive process is needed to accurately pinpoint the Central Executive function(s) that is/are most impaired by anxiety. The current study endeavoured to progress in this direction through the use of the PGNG and $n$-back tasks, and improved upon traditional studies of ACT that may be confounded by behavioural inhibition such as control of eye movements. However, the PGNG tasks combined processing efficiency measures for the set-shifting and inhibition functions, making it difficult to identify if one of these two functions are differentially affected by anxiety. Moreover, using a battery of comparable "process-pure" tasks to test the effects of anxiety on the Central Executive functions would allow researchers to discern which function(s) is/are most affected by anxiety.

The predictions of ACT are based on Miyake et al. 's [18] conclusion that the three Central Executive functions are setshifting, inhibition and updating. As has been described, the role of preattentive processes and the underlying mechanism of differential influence in the Goal-Directed and the Stimulus-Driven Attentional Systems remains unexplored in the current ACT model. Specifically, the potential role of contention scheduling and dual-task coordination reflects the need for ACT to be reviewed and extended to account for the effects of anxiety on preattentive processes. Although findings from the present study do not validate that dual-task coordination and contention scheduling form part of the attentional control system, findings from previous behavioural and neuropsychological studies are in agreement with this hypothesis. Additional investigation is required to test the proposed revision of ACT, through examining the effects of anxiety on dual-task coordination. This can be achieved through replicating the current experiment in conjunction with a dual task that involves automatic and attentive processes, such as the Stroop task. Finally, the current findings indicate future research should consider including a less mentally demanding level for the inhibition function to test the effects of anxiety on inhibition when it is not under mental strain.

\section{Implications for Attentional Control Theory}

The current study demonstrated that anxiety significantly impairs processing efficiency of the updating function as well as the inhibition and set-shifting functions. While this is consistent with Eysenck et al. 's [11] original predictions of ACT, it contradicts a recent review of the theory by Derakshan and Eysenck [12], in part due to the failure to use mentally demanding tasks to test the updating function in some of the reviewed studies.

On the experimental level, the PGNG and $n$-back tasks used in the current study produced findings that are consistent with the antisaccade paradigm studies by Ansari et al. [19] and Derakshan et al. [23], indicating these tasks as useful research tools for future investigations of the Attentional Control Theory. However it should be noted that current findings suggest a less mentally demanding level is required for the inhibition function to test the effects of anxiety on inhibition when the Central Executive under not under high demand. On the theoretical level, the current study reflected the need of ACT to consider the effects of anxiety on preattentive processes, in particular, incorporating the role of contention scheduling and dual-task coordination into the theory using the proposed model of ACT that incorporates the role of dualtask coordination and contention scheduling.

\section{CONFLICT OF INTEREST}

The authors confirm that this article content has no conflicts of interest. 


\section{ACKNOWLEDGEMENT}

Declared none.

\section{REFERENCES}

[1] Ashcraft $\mathrm{MH}$, Krause JA. Working memory, math performance, and math anxiety. Perform Bull Rev 2007; 14: 243-8.

[2] Calvo MG, Eysenck MW, Ramos PM, Jimenez A. Compensatory reading strategies in test anxiety. Anxiety Stress Coping Int J 1994; 7: 99-16.

[3] Leach J, Grffith R. Restrictions in working memory capacity during parachuting: A possible cause of 'no pull' fatalities. Appl Cogn Psychol 2008; 22: 147-57.

[4] Wilson M, Smith NC, Chattington M, Ford M, Marple-Horvat DE. The role of effort in moderating the anxiety-performance relationship: testing the prediction of processing efficiency theory in simulated rally driving. J Sport Sci 2006; 24: 1223-33.

[5] Hopko DR, McNeil DW, Lejuez CW, et al. The effects of anxious responding on mental arithmetic and lexical decision task performance. J Anxiety Disord 2003; 17; 647-65.

[6] Hopko DR, Crittendon JA, Grant E, Wilson SA. The impact of anxiety on performance IQ. Anxiety Stress Coping 2005; 18: 1735 .

[7] Eysenck MW, Calvo MG. Anxiety and performance: The processing efficiency theory. Cogn Emot 1992; 6: 409-34.

[8] Murray NP, Janelle CM. Anxiety and performance: A visual search examination of the processing efficiency theory. J Sport Exer Psychol 2003; 25: 171-87.

[9] Rapee RM. The utilization of working memory by worry. Behav Res Ther 1993; 31: 617-20.

[10] Wilson M, Smith NC, Holmes PS. The role of effort in influencing the effect of anxiety on performance: testing the conflicting predictions of processing efficiency theory and the conscious processing hypothesis. Br J Psychol 2007; 98: 411-28.

[11] Eysenck MW, Derakshan N, Santos R, Calvo MG. Anxiety and cognitive performance: Attentional control theory. Emotion 2007; 7: 336-53.

[12] Derakshan N, Eysenck MW. Anxiety, processing efficiency, and cognitive performance: new developments from attentional control theory. Eur Psychol 2009; 14, 168-76.

[13] Mogg K, Bradley BP, Dixon C, Fisher S, Twelftree H, McWilliams A. Trait anxiety, defensiveness and selective processing of threat: AN investigation using two measures o attentional bias. Pers Individ Dif 2000; 28: 1063-77.

[14] Wilson M, MacLeod C. Contrasting two accounts of anxiety-linked attentional bias: Selective attention to varying levels of stimulus threat intensity. J Abnorm Psychol 2003; 112: 212-8.

[15] Corbetta M, Shulman GL. Control of goal-directed and stimulusdriven attention in the brain. Nat Rev Neurosci 2002; 3: 201-15.

[16] Baddeley A. Exploring the central executive. Quart J Exper Psychol 1996; 49A: 5-28.

[17] Smith EE, Jonides J. Storage and executive processes in the frontal lobes. Science 1999; 283: 1657-61.

[18] Miyake A, Friedman NP, Emerson MJ, Witzki AH, Howerter A, Wager TD. The unity and diversity of executive functions and their contributions to complex 'frontal lobe' tasks: A latent variable analysis. Cogn Psychol 2000; 41: 49-100.

[19] Ansari TL, Derakshan N, Richards A. Effects of anxiety on task switching: Evidence from the mixed antisaccade task. Cogn Affect Behav Neurosci 2008; 8: 229-38.

[20] Ansari TL, Derakshan N. The neural correlates of cognitive effort in anxiety: effects on processing efficiency. Biol Psychol 2011;86: 337- 48 .
[21] Pacheco-Unguetti AP, Acosta A, Callejas L J. Attention and anxiety: Different attentional functioning under state and trait anxiety. Psychol Sci 2010; 298-304.

[22] Berggren N, Derakshan N. Attentional control deficits in trait anxiety: why you see them and why you don't. Biol Psychol 2012; (In Press)

[23] Derakshan N, Ansari TL, Hansard M, Shoker L, Eysenck MW. Anxiety, inhibition, efficiency, and effectiveness. An investigation using antisaccade task. J Exp Psychol 2009; 56: 28-55.

[24] Shackman AJ, Sarinopoulos I, Maxwell JS, Pizzagalli DA, Lavric A, Davidson RJ. Anxiety selectively disrupts visuospatial working memory. Emotion 2006; 6: 40-61.

[25] Chung S, Kwon J, Lee H, et al. Effects of high concentration oxygen administration on n-back task performance and physiological signals. Physiol Meas 2007; 28: 389-96.

[26] Langenecker SA, Bieliauskas LA, Rapport L, Zubieta J, Wilde EA, Berent S. Face emotion perception and executive functioning deficits in depression. J Clin Exp Neuropsychol 2005; 27: 320-33.

[27] Spielberger CC, Gorsuch RL, Lushene R, Vagg PR, Jacobs GA. Manual for the state-trait anxiety inventory. Palo Alto, CA: Consult Psychologists Press 1983.

[28] Langenecker SA, Zubieta J, Young EA, Akil H, Nielson KA. A task to manipulate attentional load, set-shifting, and inhibitory control: Convergent validity and test-retest reliability of the Parametric Go/No-Go Test. J Clin Exp Neuropsychol 2007; 29: 842-53.

[29] Kirchener WK. Age differences in short-term retention of rapidly changing information. J Exp Psychol 1958; 55: 352-8.

[30] Darke S. Anxiety and working memory capacity. Cogn Emot 1988; 2: 145-54.

[31] Calvo MG, Ramos P, Estevez A. Test anxiety and comprehension efficiency: The role of prior knowledge and working memory deficits. Anxiety Stress Coping 1992; 5: 125-38.

[32] Sorg BA, Witney P. The effect of trait anxiety and situational stress on working memory capacity. J Res Person 1992; 26: 235-41.

[33] Wolfe JM, Treisman A, Horowitz TS. What shall we do with the preattentive processing stage: Use it or lose it? In Third Annual Meeting of the Vision Sciences Society. Sarasota: FL. 2003.

[34] Neisser U. Cognitive psychology. New York: Appleton-CenturyCrofts 1967.

[35] Schneider W, Schiffrin RM. Controlled and automatic human information processing: I. Detection, search, and attention. Psychol Rev 1977; 84: 1-66.

[36] Norman DA, Shallice T. Attention to action: Willed and automatic control of behaviour. In Davidson RJ, Schwartz GE, Shapiro D, Eds., Consciousness and self-regulation. New York: Plenum Press. 1986; vol. 4: pp. 1-18.

[37] Collette F, Van der Linden M. Brain imaging of the central executive component of working memory. Neurosci Biobehav Rev 2002; 26: 105-25.

[38] Baddeley AD, Della S, Gray C, Papagno C, Spinnler H. Testing central executive functioning with a pencil-and-paper test. In Rabbit P. Ed. Methodology of frontal and executive function. Hove, UK: Psychology Press 1997; pp. 16-80.

[39] Fournier-Vicente S, Larigauderie P, Gaonac'h's D. More dissociations and interactions within central executive functioning: A comprehensive latent-variable analysis. Acta Psychol 2008; 129: 32-48.

[40] McDowell S, Whyte J, D'Esposito M. Working memory impairments in traumatic brain injury: Evidence from a dual-task paradigm. Neuropsychologia 1997; 35: 1341-53.

[41] Baddeley A. Working memory, thought, and action. New York: Oxford University Press 2007 